$\begin{array}{ll}\text { lon transport } & \\ \text { Q9Z0Y8 } & \text { Voltage-dependent T-type calcium channel subunit alpha-1 } \\ \text { Q24270 } & \text { Voltage-dependent calcium channel type D subunit alpha-1 } \\ \text { P91645 } & \text { Voltage-dependent calcium channel type A subunit alpha-1 } \\ \text { P27732 } & \text { Voltage-dependent L-type calcium channel subunit alpha-1D } \\ \text { Q25452 } & \text { Muscle calcium channel subunit alpha-1 } \\ \text { O42398 } & \text { Voltage-dependent L-type calcium channel subunit alpha-1S } \\ \text { Q9XES1 } & \text { Calcium-transporting ATPase 4, endoplasmic reticulum-type } \\ \text { P48768 } & \text { Sodium/calcium exchanger 2 } \\ \text { P70549 } & \text { Sodium/calcium exchanger 3 } \\ \text { O59768 } & \text { Vacuolar calcium ion transporter } \\ \text { Q01728 } & \text { Sodium/calcium exchanger 1 } \\ \text { O60312 } & \text { Probable phospholipid-transporting ATPase VA } \\ \text { Q26976 } & \text { V-type proton ATPase subunit B } \\ \text { O43520 } & \text { Probable phospholipid-transporting ATPase IC } \\ \text { P31400 } & \text { V-type proton ATPase catalytic subunit A } \\ \text { P10719 } & \text { ATP synthase subunit beta, mitochondrial } \\ \text { Q4U116 } & \text { Electrogenic sodium bicarbonate cotransporter 1 (SLC4A4) } \\ \text { Q6Q760 } & \text { Sodium leak channel non-selective protein } \\ \text { O17185 } & \text { Two pore potassium channel protein sup-9 } \\ \text { Q63472 } & \text { Potassium voltage-gated channel subfamily H member 1 } \\ \text { P17659 } & \text { Potassium voltage-gated channel subfamily A member 6 } \\ \text { Q97258 } & \text { Potassium channel subfamily T member 1 } \\ \text { P06686 } & \text { Sodium/potassium-transporting ATPase subunit alpha-2 } \\ \text { Q5REV9 } & \text { Sodium-dependent phosphate transport protein 2B (SLC34A2) } \\ \text { P48764 } & \text { Sodium/hydrogen exchanger 3 (SLC9A3) } \\ & \\ \end{array}$

coral

coral

coral

coral

coral

cora

coral

Symbiodinium

Symbiodinium

Symbiodinium

Symbiodinium coral

Symbiodinium down

Symbiodinium

coral

coral

coral

coral

other

coral

coral

cora

coral

coral

coral

other

Symbiodinium

Symbiodinium

Symbiodinium

Symbiodinium

P83299

P48284 Carbonic anhydrase 4

Q5BCC5 Carbonic anhydrase

\title{
Cell death/immunity
}

Q8WXG1 Radical S-adenosyl methionine domain-containing protein 2

Q6NZ06 Interleukin enhancer-binding factor 2 homolog

Q10471 Polypeptide $\mathrm{N}$-acetylgalactosaminyltransferase 2

P11941 Lysozyme CII

015033 Protein KIAA0317

Q29041 Ficolin-2

Q9VQQ9 Exocyst complex component 2

Q8R5F7 Interferon-induced helicase $\mathrm{C}$ domain-containing protein 1

Q5ZLI9 SAM domain and HD domain-containing protein

P04839 Cytochrome b-245 heavy chain

Q3UG20 Histone-lysine N-methyltransferase MLL5

Q86FQ0 Cytolysin Src-1

P58912 Toxin PsTX-60B

Q02817 Mucin-2

Q60803 TNF receptor-associated factor 3

Q29042 Ficolin-1

Q63772 Growth arrest-specific protein 6

P25092 Heat-stable enterotoxin receptor

P19109 ATP-dependent RNA helicase p62

Q93109 Equinatoxin-5

$\begin{array}{ll}\text { coral } & \text { down } \\ \text { coral } & \text { down } \\ \text { coral } & \text { down } \\ \text { coral } & \text { down } \\ \text { coral } & \text { down } \\ \text { coral } & \text { up } \\ \text { coral } & \text { up } \\ \text { coral } & \text { up } \\ \text { coral } & \text { up } \\ \text { coral } & \text { up } \\ \text { coral } & \text { up } \\ \text { coral } & \text { up } \\ \text { coral } & \text { up } \\ \text { coral } & \text { up } \\ \text { coral } & \text { up } \\ \text { coral } & \text { up } \\ \text { coral } & \text { up } \\ \text { other } & \text { up } \\ \text { other } & \text { up }\end{array}$

Oxidative stress

P09933 Thyroid peroxidase

Q23490 Peroxidase mlt-7

P17336 Catalase

Q970V5 Peroxiredoxin-4

Q9VEG6 Chorion peroxidase

$061235 \quad$ Catalase-2

$\begin{array}{ll}\text { coral } & \text { down } \\ \text { coral } & \text { down } \\ \text { coral } & \text { up } \\ \text { coral } & \text { up } \\ \text { coral } & \text { up } \\ \text { coral } & \text { up }\end{array}$




$\begin{array}{ll}\text { Q27487 } & \text { Peroxisomal catalase 1 } \\ \text { P11247 } & \text { Myeloperoxidase } \\ \text { O16025 } & \text { Allene oxide synthase-lipoxygenase protein } \\ \text { P22079 } & \text { Lactoperoxidase } \\ \text { O48646 } & \text { Probable phospholipid hydroperoxide glutathione peroxidase 6, mitochondrial } \\ \text { Q4PD66 } & \text { Putative heme-binding peroxidase } \\ \text { Q42592 } & \text { L-ascorbate peroxidase S, chloroplastic/mitochondrial } \\ & \\ \text { Molecular chaperones } \\ \text { Q498R3 } & \text { DnaJ homolog subfamily C member 10 } \\ \text { Q8NFJ9 } & \text { Bardet-Biedl syndrome 1 protein } \\ \text { Q99MH9 } & \text { Bardet-Biedl syndrome 2 protein homolog } \\ \text { Q8K0U4 } & \text { Heat shock 70 kDa protein 12A } \\ \text { Q9JLC8 } & \text { Sacsin } \\ \text { Q5RGU1 } & \text { Chaperone activity of bc1 complex-like, mitochondrial } \\ \text { Q54HY8 } & \text { Probable mitochondrial chaperone BCS1-A } \\ \text { Q6F2Y7 } & \text { Heat shock protein 101 } \\ \text { P19208 } & \text { Heat shock 70 kDa protein C } \\ \text { Q9US15 } & \text { Heat shock protein sti1 homolog } \\ & \\ \text { Cell homeostasis } & \\ \text { Q63120 } & \text { Canalicular multispecific organic anion transporter 1 } \\ \text { P55011 } & \text { Solute carrier family 12 member 2 } \\ \text { Q04447 } & \text { Creatine kinase B-type } \\ \text { Q920H8 } & \text { Hephaestin } \\ \text { Q5ZIH2 } & \text { Vacuolar fusion protein MON1 homolog A } \\ \text { Q8JZQ2 } & \text { AFG3-like protein 2 } \\ \text { Q5NVA2 } & \text { Thioredoxin reductase 1, cytoplasmic } \\ \text { Q9FNE2 } & \text { Glutaredoxin-C2 } \\ \text { A6H784 } & \text { Protein SCO2 homolog, mitochondrial } \\ \text { A7RQM5 } & \text { ATPase ASNA1 homolog } \\ \text { P56941 } & \text { Niemann-Pick C1 protein } \\ \text { Q6PBM1 } & \text { Glutaredoxin-related protein 5, mitochondrial } \\ \text { Q86WG5 } & \text { Myotubularin-related protein 13 } \\ \text { Q9ESR9 } & \text { ATP-binding cassette sub-family A member 2 } \\ \text { Q5E9M9 } & \text { Mitochondrial Rho GTPase 2 } \\ \text { Q9D8X1 } & \text { Copper homeostasis protein cutC homolog } \\ \text { O95881 } & \text { Thioredoxin domain-containing protein 12 } \\ \text { Q8NBS9 } & \text { Thioredoxin domain-containing protein 5 } \\ \text { P10719 } & \text { ATP synthase subunit beta, mitochondrial } \\ \text { O95477 } & \text { ATP-binding cassette sub-family A member 1 } \\ \text { P83877 } & \text { Thioredoxin-like protein 4A } \\ & \end{array}$

Molecular chaperones

Response to ER stress

$\begin{array}{ll}\text { Q32LH7 } & \text { Cytochrome b5 reductase 4 } \\ \text { P54399 } & \text { Protein disulfide-isomerase } \\ \text { O22263 } & \text { Protein disulfide-isomerase like 2-1 } \\ \text { Q67IX6 } & \text { Protein disulfide isomerase-like 1-4 } \\ \text { Q17967 } & \text { Protein disulfide-isomerase 1 } \\ \text { P38657 } & \text { Protein disulfide-isomerase A3 } \\ \text { Q29RV1 } & \text { Protein disulfide-isomerase A4 } \\ \text { O94726 } & \text { ER degradation-enhancing alpha-mannosidase-like protein 1 } \\ \text { P09103 } & \text { Protein disulfide-isomerase } \\ \text { P34329 } & \text { Probable protein disulfide-isomerase A4 }\end{array}$

\section{Calcium signaling/homeostasis}

\begin{tabular}{|c|c|c|c|}
\hline Q64143 & Phosphatidylinositol 3-kinase regulatory subunit gamma & coral & down \\
\hline Q8CHJ1 & Phosphatidylinositol glycan anchor biosynthesis class U protein & coral & down \\
\hline P29994 & Inositol 1,4,5-trisphosphate receptor type 1 & coral & up \\
\hline Q63269 & Inositol 1,4,5-trisphosphate receptor type 3 & coral & up \\
\hline Q14571 & Inositol 1,4,5-trisphosphate receptor type 2 & coral & up \\
\hline P29993 & Inositol 1,4,5-trisphosphate receptor & coral & up \\
\hline A2A891 & Calmodulin-binding transcription activator 1 & coral & up \\
\hline Q6DEH3 & Calcium/calmodulin-dependent protein kinase type II delta 1 chain & coral & up \\
\hline Q9Y217 & 1-phosphatidylinositol-3-phosphate 5-kinase & coral & up \\
\hline P51432 & 1-phosphatidylinositol-4,5-bisphosphate phosphodiesterase beta-3 & coral & up \\
\hline A4IID4 & Phosphatidylinositol 4-kinase beta & coral & up \\
\hline Q22908 & Ras and EF-hand domain-containing protein homolog & coral & up \\
\hline A0JP43 & EF-hand calcium-binding domain-containing protein 5 & coral & up \\
\hline A2ARV4 & Low-density lipoprotein receptor-related protein 2 & coral & up \\
\hline Q32TF8 & EF-hand domain-containing family member $\mathrm{C} 2$ & other & down \\
\hline 096102 & Calmodulin & other & up \\
\hline \multicolumn{4}{|c|}{ Wnt Signalling } \\
\hline P33945 & Protein Wnt-5b & coral & down \\
\hline 042280 & Protein Wnt-9a & coral & up \\
\hline P51028 & Protein Wnt-8a & coral & up \\
\hline
\end{tabular}




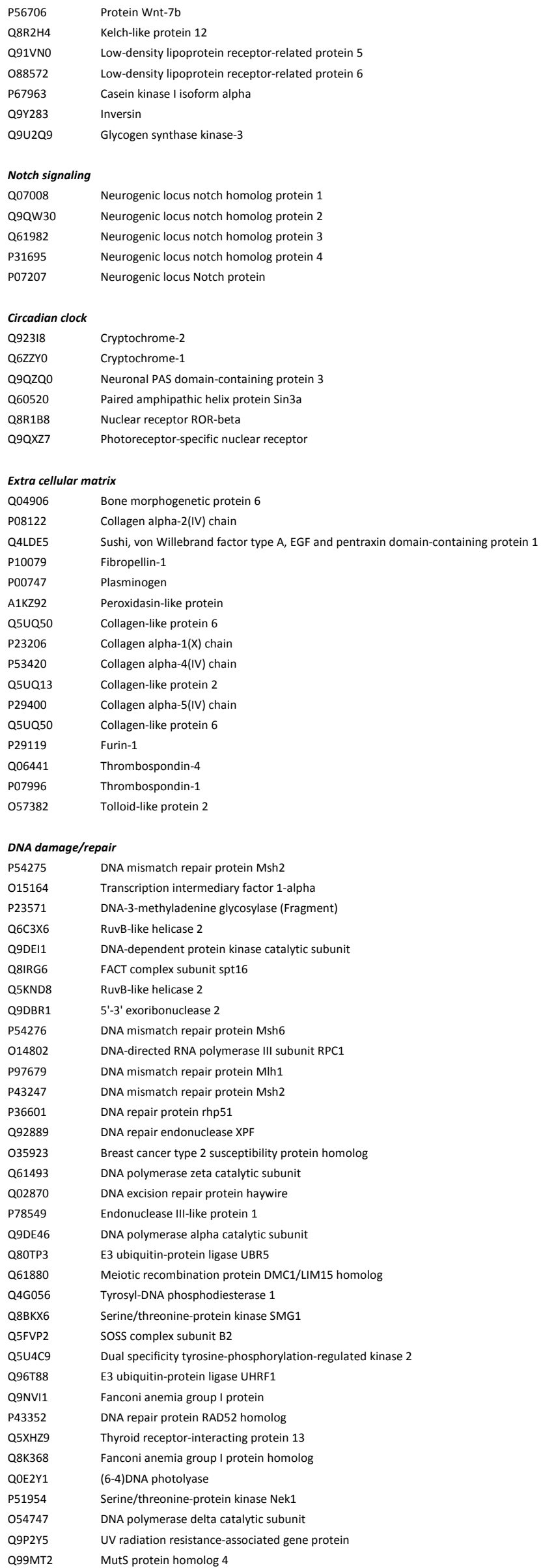

Circadian clock

Q92318 Cryptochrome-2

Q6ZZYO Cryptochrome-1

Q9QZQ0 Neuronal PAS domain-containing protein 3

Q60520 Paired amphipathic helix protein Sin3a

Q8R1B8 Nuclear receptor ROR-beta

Q90XZ7 Photoreceptor-specific nuclear receptor

Extra cellular matrix

Q04906 Bone morphogenetic protein

P08122 Collagen alpha-2(IV) chain

Q4LDE5 Sushi, von Willebrand factor type A, EGF and pentraxin domain-containing protein 1

P10079 Fibropellin-1

P00747 Plasminogen

A1KZ92 Peroxidasin-like protein

Q5UQ50 Collagen-like protein 6

P23206 Collagen alpha- $1(X)$ chain

P53420 Collagen alpha-4(IV) chain

Q5UQ13 Collagen-like protein 2

P29400 Collagen alpha-5(IV) chain

Q5UQ50 Collagen-like protein 6

P29119 Furin-1

Q06441 Thrombospondin-4

P07996 Thrombospondin-1

057382 Tolloid-like protein 2

DNA damage/repair

$015164 \quad$ Transcription intermediary factor 1-alpha

P23571 DNA-3-methyladenine glycosylase (Fragment)

Q6C3X6 RuvB-like helicase 2

Q9DEI1 DNA-dependent protein kinase catalytic subunit

Q8IRG6 FACT complex subunit spt16

Q5KND8 RuvB-like helicase 2

Q9DBR1 5'-3' exoribonuclease 2

P54276 DNA mismatch repair protein Msh6

014802 DNA-directed RNA polymerase III subunit RPC1

P97679 DNA mismatch repair protein Mlh1

P43247 DNA mismatch repair protein Msh2

P36601 DNA repair protein rhp51

Q92889 DNA repair endonuclease XPF

035923 Breast cancer type 2 susceptibility protein homolog

Q61493 DNA polymerase zeta catalytic subunit

Q02870 DNA excision repair protein haywire

P78549 Endonuclease III-like protein 1

Q9DE46 DNA polymerase alpha catalytic subunit

Q80TP3 E3 ubiquitin-protein ligase UBR5

Q61880 Meiotic recombination protein DMC1/LIM15 homolog

Q4G056 Tyrosyl-DNA phosphodiesterase 1

Q8BKX6 Serine/threonine-protein kinase SMG1

Q5FVP2 SOSS complex subunit B2

Q5U4C9 Dual specificity tyrosine-phosphorylation-regulated kinase 2

Q96T88 E3 ubiquitin-protein ligase UHRF1

Q9NVI1 Fanconi anemia group I protein

P43352 DNA repair protein RAD52 homolog

Q5XHZ9 Thyroid receptor-interacting protein 13

Q8K368 Fanconi anemia group I protein homolog

QOE2Y1 (6-4)DNA photolyase

P51954 Serine/threonine-protein kinase Nek1

054747 DNA polymerase delta catalytic subunit

Q9P2Y5 UV radiation resistance-associated gene protein

Q99MT2 MutS protein homolog 4

$\begin{array}{ll}\text { coral } & \text { down } \\ \text { coral } & \text { down } \\ \text { coral } & \text { down } \\ \text { coral } & \text { down } \\ \text { coral } & \text { down }\end{array}$

$\begin{array}{ll}\text { coral } & \text { up } \\ \text { coral } & \text { up } \\ \text { coral } & \text { up } \\ \text { coral } & \text { up } \\ \text { coral } & \text { up } \\ \text { coral } & \text { up }\end{array}$

$\begin{array}{ll}\text { coral } & \text { dow } \\ \text { coral } & \text { dow } \\ \text { coral } & \text { dow } \\ \text { coral } & \text { dow } \\ \text { coral } & \text { dow } \\ \text { coral } & \text { up } \\ \text { coral } & \text { up } \\ \text { coral } & \text { up } \\ \text { coral } & \text { up } \\ \text { coral } & \text { up } \\ \text { coral } & \text { up } \\ \text { coral } & \text { up } \\ \text { coral } & \text { up } \\ \text { coral } & \text { up } \\ \text { coral } & \text { up } \\ \text { coral } & \text { up }\end{array}$

$\begin{array}{ll}\text { coral } & \text { down } \\ \text { coral } & \text { down } \\ \text { coral } & \text { down } \\ \text { coral } & \text { down } \\ \text { coral } & \text { down } \\ \text { coral } & \text { down } \\ \text { coral } & \text { down } \\ \text { coral } & \text { down } \\ \text { coral } & \text { up } \\ \text { coral } & \text { up } \\ \text { coral } & \text { up } \\ \text { coral } & \text { up } \\ \text { coral } & \text { up } \\ \text { coral } & \text { up } \\ \text { coral } & \text { up } \\ \text { coral } & \text { up } \\ \text { coral } & \text { up } \\ \text { coral } & \text { up } \\ \text { coral } & \text { up } \\ \text { coral } & \text { up } \\ \text { coral } & \text { up } \\ \text { coral } & \text { up } \\ \text { coral } & \text { up } \\ \text { coral } & \text { up } \\ \text { coral } & \text { up } \\ \text { coral } & \text { up } \\ \text { coral } & \text { up } \\ \text { coral } & \text { up } \\ \text { coral } & \text { up } \\ \text { coral } & \text { up } \\ \text { coral } & \\ \text { coral } & \text { up } \\ \text { coral } & \text { coral } \\ \text { coral } & \end{array}$




\begin{tabular}{|c|c|}
\hline Q9LEF5 & FACT complex subunit SSRP1 \\
\hline Q6ZQFo & DNA topoisomerase 2-binding protein 1 \\
\hline 015457 & MutS protein homolog 4 \\
\hline Q5DU05 & Centrosomal protein of $164 \mathrm{kDa}$ \\
\hline Q1XDF4 & ATP-dependent Clp protease ATP-binding subunit clpA homolog \\
\hline P29295 & Casein kinase I homolog HRR25 \\
\hline Q5BBV9 & RuvB-like helicase 1 \\
\hline Q99PKO & Pre-mRNA-splicing factor SYF1 \\
\hline Q59LR2 & Serine/threonine-protein kinase MEC1 \\
\hline Q9SL02 & DNA repair protein RAD50 \\
\hline \multicolumn{2}{|c|}{ Proteolysis } \\
\hline Q63570 & $26 \mathrm{~S}$ protease regulatory subunit $6 \mathrm{~B}$ \\
\hline P19205 & Acylamino-acid-releasing enzyme \\
\hline Q8JZQ2 & AFG3-like protein 2 \\
\hline Q9NR09 & Baculoviral IAP repeat-containing protein 6 \\
\hline Q9JLG8 & Calpain-15 \\
\hline Q9נJ31 & Cullin-5 \\
\hline Q9D1A2 & Cytosolic non-specific dipeptidase \\
\hline Q616G8 & E3 ubiquitin-protein ligase HECW2 \\
\hline Q6GNY1 & E3 ubiquitin-protein ligase mib1 \\
\hline Q6E2N3 & E3 ubiquitin-protein ligase TRIM33 \\
\hline P42785 & Lysosomal Pro- $X$ carboxypeptidase \\
\hline Q7ZVX6 & NEDD8-activating enzyme E1 catalytic subunit \\
\hline P79953 & Ovochymase-2 \\
\hline P26262 & Plasma kallikrein \\
\hline A7SXZ6 & Probable tRNA threonylcarbamoyladenosine biosynthesis protein osgep \\
\hline P34676 & Prolyl carboxy peptidase like protein 5 \\
\hline 070196 & Prolyl endopeptidase \\
\hline Q9Z1k5 & Protein ariadne- 1 homolog \\
\hline 076924 & Protein ariadne-2 \\
\hline 015033 & Protein KIAA0317 \\
\hline Q9HB40 & Retinoid-inducible serine carboxypeptidase \\
\hline Q5U405 & Transmembrane protease serine 13 \\
\hline Q7Z410 & Transmembrane protease serine 9 \\
\hline Q5R761 & U4/U6.U5 tri-snRNP-associated protein 2 \\
\hline Q5RCD3 & Ubiquitin carboxyl-terminal hydrolase 4 \\
\hline Q5R407 & Ubiquitin carboxyl-terminal hydrolase 5 \\
\hline Q70EK9 & Ubiquitin carboxyl-terminal hydrolase 51 \\
\hline Q9JJZ4 & Ubiquitin-conjugating enzyme E2 J1 \\
\hline AOPJN4 & Ubiquitin-conjugating enzyme E2Q-like protein 1 \\
\hline Q9D906 & Ubiquitin-like modifier-activating enzyme ATG7 \\
\hline Q9YMP9 & Viral cathepsin \\
\hline Q8CEG8 & Ubiquitin carboxyl-terminal hydrolase 27 \\
\hline Q7KN62 & Transitional endoplasmic reticulum ATPase TER94 \\
\hline P28840 & Neuroendocrine convertase 1 \\
\hline P97435 & Enteropeptidase \\
\hline Q8BU03 & Periodic tryptophan protein 2 homolog \\
\hline 015072 & A disintegrin and metalloproteinase with thrombospondin motifs 3 \\
\hline Q9UKP4 & A disintegrin and metalloproteinase with thrombospondin motifs 7 \\
\hline A4IHP4 & Cullin-3 \\
\hline Q0P4M4 & Cytosolic carboxypeptidase 2 \\
\hline Q96MI9 & Cytosolic carboxypeptidase 4 \\
\hline Q8CF97 & Deubiquitinating protein VCIP135 \\
\hline 070260 & E3 SUMO protein ligase PIAS3 \\
\hline Q69ZR2 & E3 ubiquitin-protein ligase HECTD1 \\
\hline Q4U2R1 & E3 ubiquitin-protein ligase HERC2 \\
\hline Q7TMY8 & E3 ubiquitin-protein ligase HUWE1 \\
\hline Q6ZQ89 & E3 ubiquitin-protein ligase MARCH6 \\
\hline Q5ZIJ9 & E3 ubiquitin-protein ligase MIB2 \\
\hline Q5R7T5 & E3 ubiquitin-protein ligase NRDP1 \\
\hline Q9R1A8 & E3 ubiquitin-protein ligase RFWD2 \\
\hline Q2TL32 & E3 ubiquitin-protein ligase UBR4 \\
\hline Q80TP3 & E3 ubiquitin-protein ligase UBR5 \\
\hline Q7TPK1 & E3 ubiquitin-protein ligase UHRF1 \\
\hline P42893 & Endothelin-converting enzyme 1 \\
\hline Q8VBV4 & F-box/WD repeat-containing protein 7 \\
\hline Q9R1K5 & Fizzy-related protein homolog \\
\hline Q9CXY9 & GPI-anchor transamidase \\
\hline P35559 & Insulin-degrading enzyme \\
\hline Q1L5Z9 & LON peptidase $\mathrm{N}$-terminal domain and RING finger protein 2 \\
\hline Q9D4H7 & LON peptidase $\mathrm{N}$-terminal domain and RING finger protein 3 \\
\hline Q9Y3Q0 & $\mathrm{N}$-acetylated-alpha-linked acidic dipeptidase 2 \\
\hline Q9UQQ1 & $\mathrm{N}$-acetylated-alpha-linked acidic dipeptidase-like protein \\
\hline Q8R554 & OTU domain-containing protein 7A \\
\hline Q5K2P9 & Polyserase-2 \\
\hline Q14669 & Probable E3 ubiquitin-protein ligase TRIP12 \\
\hline
\end{tabular}

$\begin{array}{ll}\text { coral } & \text { up } \\ \text { coral } & \text { up } \\ \text { other } & \text { dow } \\ \text { Symbiodinium } & \text { dow } \\ \text { Symbiodinium } & \text { dow } \\ \text { Symbiodinium } & \text { up } \\ \text { Symbiodinium } & \text { up } \\ \text { Symbiodinium } & \text { up } \\ \text { Symbiodinium } & \text { up } \\ \text { Symbiodinium } & \text { up }\end{array}$




\begin{tabular}{|c|c|}
\hline P70398 & Probable ubiquitin carboxyl-terminal hydrolase FAF-X \\
\hline Q8VDW4 & Probable ubiquitin-conjugating enzyme E2 W \\
\hline Q61139 & Proprotein convertase subtilisin/kexin type 7 \\
\hline Q16651 & Prostasin \\
\hline Q8T4E1 & Putative GPI-anchor transamidase \\
\hline Q9HBA9 & Putative $\mathrm{N}$-acetylated-alpha-linked acidic dipeptidase \\
\hline Q9FHW7 & SKP1-like protein 1B \\
\hline A0JMG1 & Speckle-type POZ protein-like B \\
\hline Q9Z1F9 & SUMO-activating enzyme subunit 2 \\
\hline 054928 & Suppressor of cytokine signaling 5 \\
\hline Q9UPU5 & Ubiquitin carboxyl-terminal hydrolase 24 \\
\hline Q70CQ4 & Ubiquitin carboxyl-terminal hydrolase 31 \\
\hline Q8NFAO & Ubiquitin carboxyl-terminal hydrolase 32 \\
\hline Q6ZQ93 & Ubiquitin carboxyl-terminal hydrolase 34 \\
\hline Q8BUM9 & Ubiquitin carboxyl-terminal hydrolase 43 \\
\hline P52483 & Ubiquitin-conjugating enzyme E2 E3 \\
\hline Q9NPD8 & Ubiquitin-conjugating enzyme E2 T \\
\hline P55115 & Zinc metalloproteinase nas-15 \\
\hline Q8VZS9 & Protein FIZZY-RELATED 1 \\
\hline Q9DBI0 & Transmembrane protease serine 6 \\
\hline P46472 & 265 protease regulatory subunit 7 \\
\hline Q1XDF9 & ATP-dependent zinc metalloprotease FtsH \\
\hline Q8HOW1 & Chloroplast processing peptidase \\
\hline P22412 & Dipeptidase 1 \\
\hline Q7KUT2 & Lon protease homolog, mitochondrial \\
\hline B8J198 & Lon protease \\
\hline Q8L3Z8 & Protein FIZZY-RELATED 2 \\
\hline Q8LPL5 & Protein FIZZY-RELATED 3 \\
\hline Q42384 & Protein pleiotropic regulatory locus 1 \\
\hline Q9FF66 & Ubiquitin-conjugating enzyme E2 22 \\
\hline Q9LL3 & Presequence protease 1 , chloroplastic/mitochondrial \\
\hline Q5PQY6 & Lon protease homolog 2, peroxisomal \\
\hline Q8VY06 & Presequence protease 2 , chloroplastic/mitochondrial \\
\hline Q4FZT9 & $26 \mathrm{~S}$ proteasome non-ATPase regulatory subunit 2 \\
\hline P72991 & ATP-dependent zinc metalloprotease Fts 3 \\
\hline A6QBN8 & ATP-dependent zinc metalloprotease FtsH \\
\hline Q6GPU1 & Cysteine protease ATG4A \\
\hline 074445 & Probable 265 protease subunit rpt 4 \\
\hline Q6J1Y9 & Ubiquitin carboxyl-terminal hydrolase 19 \\
\hline \multicolumn{2}{|c|}{ Cytoskeletal interactions } \\
\hline P27619 & Dynamin \\
\hline P46824 & Kinesin light chain \\
\hline Q7KN62 & Transitional endoplasmic reticulum ATPase TER94 \\
\hline P52275 & Tubulin beta- 2 chain \\
\hline 073787 & Gamma-tubulin complex component 3 homolog \\
\hline Q7TSG1 & Centrosomal protein of $120 \mathrm{kDa}$ \\
\hline P38650 & Cytoplasmic dynein 1 heavy chain 1 \\
\hline Q27802 & Cytoplasmic dynein 2 heavy chain 1 \\
\hline Q14008 & Cytoskeleton-associated protein 5 \\
\hline Q6PCJ1 & Dynactin subunit 1 \\
\hline P23098 & Dynein beta chain, ciliary \\
\hline Q9P2D7 & Dynein heavy chain 1 , axonemal \\
\hline Q8IVF4 & Dynein heavy chain 10 , axonemal \\
\hline Q96DT5 & Dynein heavy chain 11 , axonemal \\
\hline Q923J6 & Dynein heavy chain 12 , axonemal \\
\hline Q9UFH2 & Dynein heavy chain 17 , axonemal \\
\hline Q9P225 & Dynein heavy chain 2 , axonemal \\
\hline Q8TD57 & Dynein heavy chain 3 , axonemal \\
\hline Q8TE73 & Dynein heavy chain 5 , axonemal \\
\hline Q9C0G6 & Dynein heavy chain 6 , axonemal \\
\hline Q8WXX0 & Dynein heavy chain 7 , axonemal \\
\hline Q91XQ0 & Dynein heavy chain 8 , axonemal \\
\hline Q9NYC9 & Dynein heavy chain 9 , axonemal \\
\hline P37276 & Dynein heavy chain, cytoplasmic \\
\hline Q9TVM2 & Exportin-1 \\
\hline Q92845 & Kinesin-associated protein 3 \\
\hline P46871 & Kinesin-II 95 kDa subunit \\
\hline Q9GYZO & Kinesin-like protein KIF15 \\
\hline 035787 & Kinesin-like protein KIF1C \\
\hline Q9QXL1 & Kinesin-like protein KIF21B \\
\hline Q52KG5 & Kinesin-like protein KIF26A \\
\hline Q7TNC6 & Kinesin-like protein KIF26B \\
\hline P28739 & Kinesin-like protein klpA \\
\hline Q63356 & Myosin-le \\
\hline Q29P71 & Myosin-Vlla \\
\hline Q6PIF6 & Myosin-VIIb \\
\hline
\end{tabular}




$\begin{array}{ll}\text { Q5ZMV2 } & \text { Spindle assembly abnormal protein } 6 \text { homolog } \\ \text { Q62868 } & \text { Rho-associated protein kinase 2 } \\ \text { Q8WQ47 } & \text { Tubulin alpha chain } \\ \text { O44388 } & \text { Tubulin beta chain } \\ \text { Q9D6F9 } & \text { Tubulin beta-4 chain } \\ \text { Q9UJT0 } & \text { Tubulin epsilon chain } \\ \text { Q14008 } & \text { Cytoskeleton-associated protein } 5 \\ \text { Q9P2E2 } & \text { Kinesin-like protein KIF17 } \\ \text { Q9U2Q9 } & \text { Glycogen synthase kinase-3 } \\ \text { P29295 } & \text { Casein kinase I homolog HRR25 } \\ \text { Q9QXL2 } & \text { Kinesin-like protein KIF21A } \\ \text { P23257 } & \text { Tubulin gamma-1 chain }\end{array}$

Cell cycle/cell proliferation regulation

Q8CG48 Structural maintenance of chromosomes protein 2

Q9Y236 Oxidative stress-induced growth inhibitor 2

Q61127 NGFI-A-binding protein 2

Q28D84 Histone-lysine N-methyltransferase EZH2

Q28DT7 Polycomb protein eed

Q641W4 Replication factor $\mathrm{C}$ subunit 2

Q9DEI1 DNA-dependent protein kinase catalytic subunit

P97386 DNA ligase 3

Q64213 Splicing factor 1

P0C279 Cytoplasmic polyadenylation element-binding protein 1

Q61880 Meiotic recombination protein DMC1/LIM15 homolog

P38129 Transcription initiation factor TFIID subunit 5

Q7TSG1 Centrosomal protein of $120 \mathrm{kDa}$

Q66113 Growth factor receptor-bound protein 2

Q6KCD5 Nipped-B-like protein

Q00526 Cyclin-dependent kinase 3

P50532 Structural maintenance of chromosomes protein 4

P50533 Structural maintenance of chromosomes protein 2

Q5ZMV2 Spindle assembly abnormal protein 6 homolog

P62296 Abnormal spindle-like microcephaly-associated protein homolog

GAS2-like protein 1

$\begin{array}{ll}\text { Q8JZP9 } & \text { GAS2-like protein } 1 \\ \text { Q8VIG3 } & \text { Radial spoke head } 1 \text { homolog }\end{array}$

P42128 Forkhead box protein K1

Q9U2Q9 Glycogen synthase kinase-3

P97310 DNA replication licensing factor MCM2

Q80YP0 Cyclin-dependent kinase 3

P23573 Cell division control protein 2 cognate

P30665 DNA replication licensing factor MCM4

Q5DU05 Centrosomal protein of $164 \mathrm{kDa}$

Q9UW86 Serine/threonine-protein phosphatase PP1

Q99KE1 NAD-dependent malic enzyme, mitochondrial

Q2KIZ3 Methylmalonyl-CoA epimerase, mitochondrial

Q8BHF7 CDP-diacylglycerol--glycerol-3-phosphate 3-phosphatidyltransferase, mitochondrial

Q148G5 Proline dehydrogenase 1, mitochondrial

Q08D64 ATP-binding cassette sub-family B member 6, mitochondrial

Q5Z160 3-hydroxyisobutyryl-CoA hydrolase, mitochondrial

Q5RFN2 Methylmalonyl-CoA mutase, mitochondrial

B1P1W2 Succinate dehydrogenase assembly factor 2, mitochondrial

Q3SZ18 Isovaleryl-CoA dehydrogenase, mitochondrial

P70079 Creatine kinase U-type, mitochondrial

Q8QZS1 3-hydroxyisobutyryl-CoA hydrolase, mitochondrial

Q1JPD3 D-2-hydroxyglutarate dehydrogenase, mitochondrial

P50671 Cytochrome $c$ oxidase subunit 1 (Fragment)

Q96190 Cytochrome $\mathrm{c}$ oxidase subunit 2

BOU3F2 Glycerol kinase

Q9TTS3 Acetyl-CoA carboxylase 1

P12785 Fatty acid synthase

Q68J42 Hormone-sensitive lipase

Q9UH92 Max-like protein $X$

P38652 Phosphoglucomutase-1

P79303 UTP--glucose-1-phosphate uridylyltransferase

P17625 Glycogen [starch] synthase, liver

A6QLU1 Glycerol-3-phosphate dehydrogenase, mitochondrial

$\begin{array}{ll}\text { coral } & \text { down } \\ \text { coral } & \text { down } \\ \text { coral } & \text { down } \\ \text { coral } & \text { down } \\ \text { coral } & \text { down } \\ \text { coral } & \text { down } \\ \text { coral } & \text { down } \\ \text { coral } & \text { down } \\ \text { coral } & \text { down } \\ \text { coral } & \text { down } \\ \text { coral } & \text { down } \\ \text { coral } & \text { down } \\ \text { coral } & \text { down } \\ \text { coral } & \text { down } \\ \text { coral } & \text { down } \\ \text { coral } & \text { down } \\ \text { coral } & \text { down } \\ \text { coral } & \text { down } \\ \text { coral } & \text { down } \\ \text { coral } & \text { down } \\ \text { coral } & \text { down } \\ \text { coral } & \text { down } \\ \text { coral } & \text { down } \\ \text { coral } & \text { down } \\ \text { coral } & \text { down } \\ \text { coral } & \text { down } \\ \text { coral } & \text { down } \\ \text { coral } & \text { down } \\ \text { coral } & \text { up }\end{array}$


Presequence protease 1 , chloroplastic/mitochondrial 